\title{
3D MODELLING OF CHUA'S CIRCUIT BOUNDARY SURFACE
}

\author{
Branislav SOBOTA \\ Department of Computers and Informatics, Faculty of Electrical Engineering and Informatics, \\ Technical University of Košice, Letná 9, 04200 Košice, Slovak Republic, tel.: +421 55602 2563, e-mail: branislav.sobota@tuke.sk
}

\begin{abstract}
In this paper is presented the 3D model of Chua's circuit boundary surfaces. This represents the boundary between the two attractors - chaos and a stable limit cycle. In the first part is described short history of Chua's circuit and sequential circuits boundary surface modelling and visualization. Second part describes $3 D$ modeling of boundary surface. Three dimensional model of boundary surface is created by appropriate combination lot of $2 D$ cross-sections in surface $u 1=c o n s t$ using triangulation. The modelling and visualization of boundary surface represents modelling and visualization of large graphical data set and is timeconsuming process.
\end{abstract}

Keywords: computer graphics, 3D modelling, boundary surface, Chua's circuit, 2D cross-sections, triangulation

\section{INTRODUCTION}

An important part of the analysis of circuits, characterized by at least two attractors is a boundary surface (BS). If we know it, we can comment on the size of the corresponding attractive areas, options to control the circuit, or the circuit resistance against disturbances. In the case of $2 \mathrm{D}$ BS - the border separating the attractors from each other is the line - separatrix, but in 3D-BS is the surface. Calculation of BS makes high demands not only to PC hardware used (especially CPU), but in the case of 3D view also to PC graphics. Despite the mentioned demands to hardware $3 \mathrm{D}$ viewing of $\mathrm{BS}$ provides an advantage that allows accumulating dozens of partial 2D cross-sections [1], [2] into the single image [3]. Informative value of the picture may thus increase not only by viewing the BS, but also by possibility of manipulation with 3D object - BS. If the proposed software will add to the displayed 3D object corresponding trajectories, or $I V$ characteristics, illustration figure will increase. If the proposed software will be added to the displayed 3D object and the trajectory, and $I V$ characteristics, illustration the figure will increase again.

\section{BOUNDARY SURFACE}

The first curves illustrating the dividing state plane among corresponding attractors are shown in [4][5][6]. These curves were calculated and plotted using graphicnumerical methods developed by the author of those works. Later, the introduction of computers significantly accelerated calculation of separatrix and trajectories for analysed circuits [7][8][9] also in 3D space $(m=3)$, or $m>3$ [10] [11]. Probably the first display of the BS morphology in 3D space with help of Matlab was showed in [12]. There was analysed ternary memory, where surprisingly began to appear undesirable stable limit cycle (SLC). It is a space between BS2 and BS3, which arisen from splitting up the BS that separated the second and third stable state (logic level) of the ternary memory, Fig. 1. Fig. 1 shows BS2 separating attraction area for the second stable state (S2) from the attraction area for SLC LC1. BS3, in turn, separates the LC1 from S3 (the third stable state). BS1 classically separates S1 from S2.

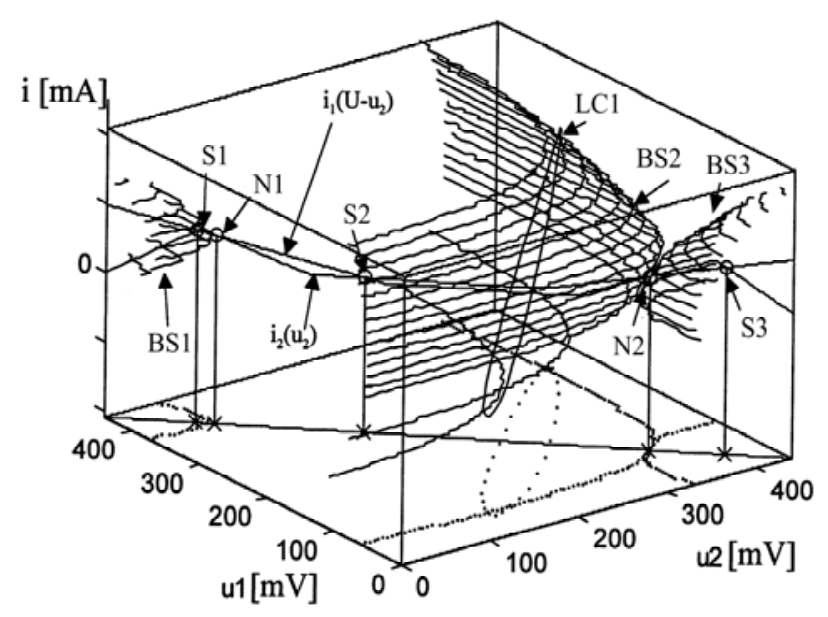

Fig. 1 3D view of BS using contour lines

Like in [12], the Matlab was used for graphically friendlier displaying of the BS again for ternary memory, but in case where in the addition to the three static attractors (S1, S2, S3), there are three other undesirable dynamic attractors SLC (LC1, LC2, LC3). Dividing the state space into the attraction areas for corresponding attractors in the plane $x_{1}, x_{2}$ in 3D space shows Fig. 2. Case where the memory is not characterized by oscillations is illustrated in Figure 3. Mentioned visualization the BS was realized using functions Surface and Mesh [3]. Symbols AO indicate the attraction areas for the individual attractors. As is shown in Fig. 2a, although BS is quite rugged, still consists of relatively simple closed curves (Fig. 2a, Fig. 3a), whose contours can be linked to arrive at a 3D BS showed in Fig. 2b and 3b.

\section{3D MODELLING OF BS}

In order to visualize BS for similar Chua's circuit BS using the parameters listed in [13] complication occurred to the identification of individual points to create surfaces in 3D. It happened because of the sudden change in the shape of BS cross-section formed "sharp teeth". Some from 300 calculated BS cross-sections illustrates Fig. 4. BS visualization only by contour lines, like in Fig. 1, has been shown in [14]. This is a cross-section in the plane 
$i, u_{2}$ which coordinate system begins in the middle of the picture, and $i<-27,27>\mathrm{A}$ and $u_{2}<-9 ; 9>\mathrm{V}$.
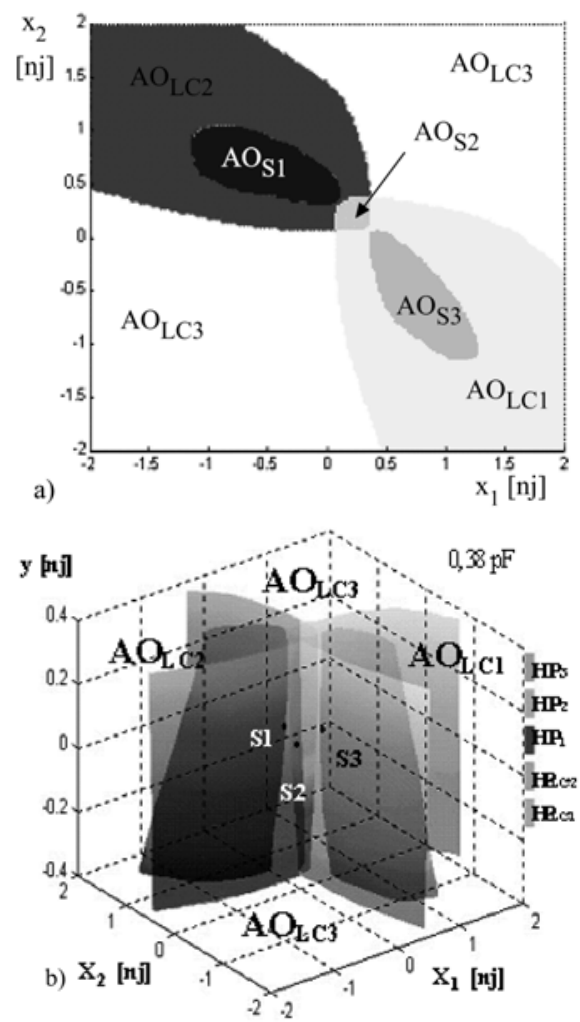

Fig. 2 BS in a normalized state space for the existence of three SLC a) cross-section the BS in $x_{1}, x_{2}$ plane b) 3D model of BS
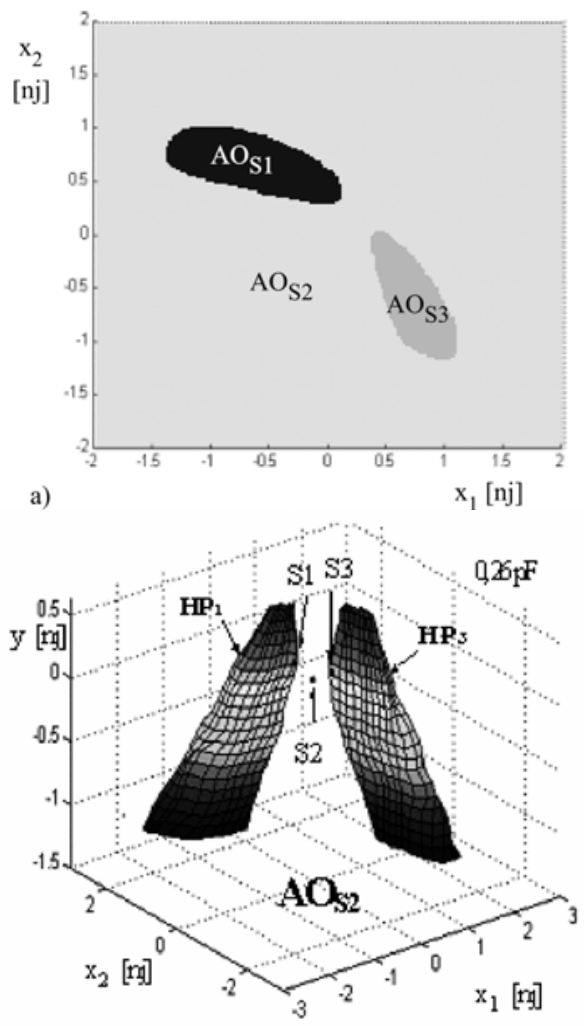

b)

Fig. 3 BS in a normalized state space in the absence of three SLC a) cross-section the BS in $x_{1}, x_{2}$ plane b) $3 \mathrm{D}$ model of BS
Number listed in the top right corner indicates the $u_{I}$ value [V] for which the BS cross-section was calculated. Each cross-section is created from net of $440 \times 440$ points (initial conditions), which represents 193600 calculated trajectories. Black, respectively white section represents attraction area for chaotic attractor or SLC. To create 3D model of BS at work [15] were used works knowledge $[16][17][18][19]$ and was used procedure described below.

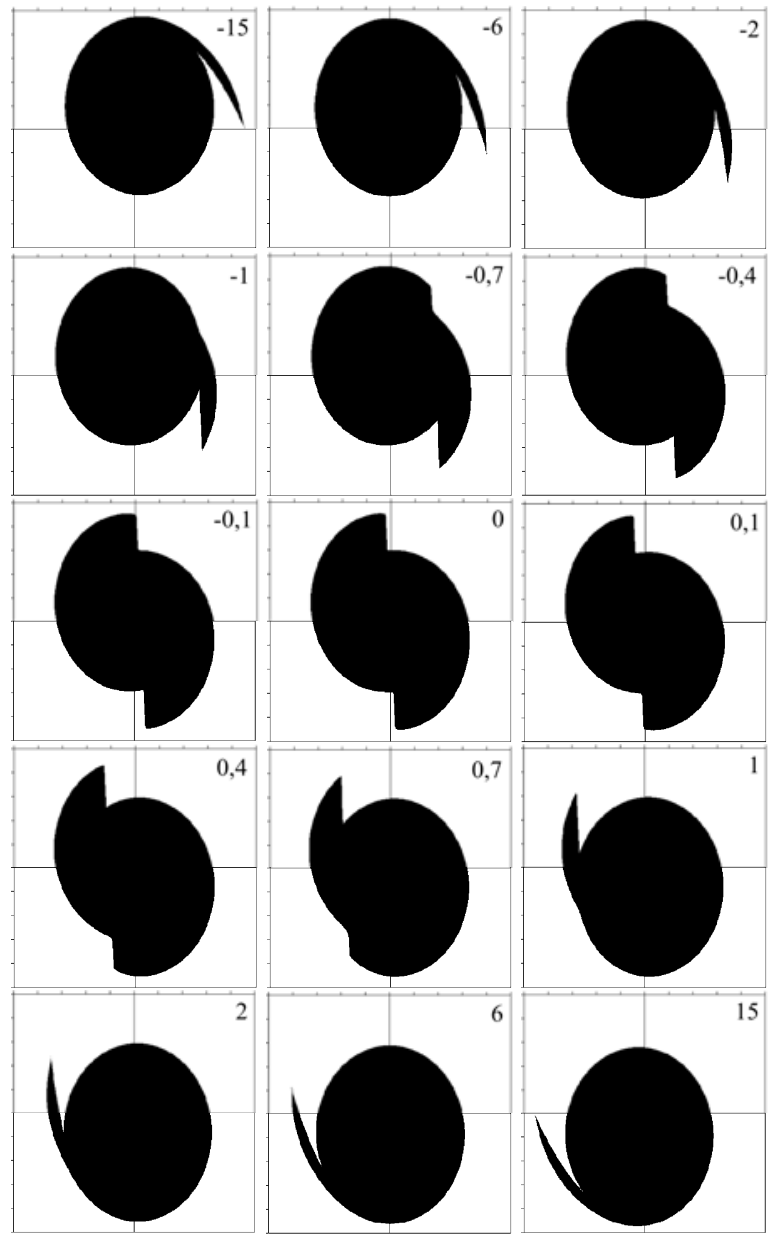

Fig. 4 BS cross-sections of Chua's circuit in $i, u_{2}$ plane for $u_{1}$ listed in the top right corner

Given the BS cross-sections complex shape crosssection was (BS contour) divided into four segments four separated curves abiding contour elevation respectively descent.

Their endpoints connected with next contour endpoints. This created rectangles that were lapping gaps between two contours. In general are during wire model construction used triangle primitives, which more precisely define models shape (4). It was necessary to find a suitable triangulation procedure between points of following contours so model will not include any holes (places in wire model where triangle absents) so the final model will represent natural model as close as possible.

If BS cross-sections around $0 \mathrm{~V}$ cannot be clearly divided into an equal segments number because of different contour shape characteristic then another way is needed. As the following two contours hasn't already been divided into equal segments number, it was impossible to determine corresponding segments contour. Moreover 
segments shape could be very different which will cause absolutely abnormal shape of reconstructed surface. It this case, it was clear to find another much more universal triangulation and reconstruction way.

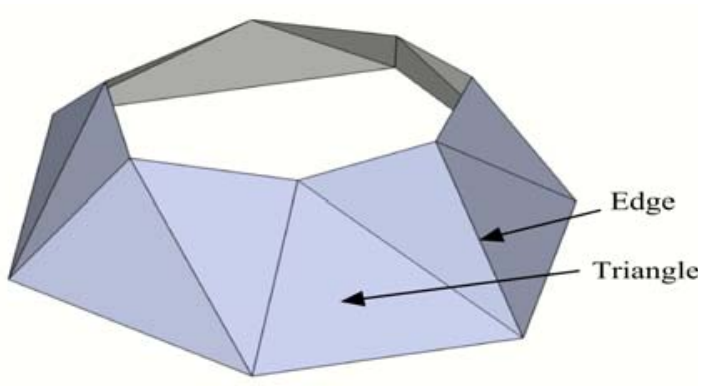

Fig. 5 Example of space filling between two contours using triangle primitives

Before the reconstruction the two steps are necessary. The first step is the contours detection in cross-sections because cross-sections are represented by a specific internal format and save the contours into separate files. The second essential step is to determine the contour perimeter points order in one direction. A possible solution of the two aforementioned steps have been using marching squares algorithm. This algorithm is a marching cubes algorithm simplification. This algorithm can be used with good results in classic raster images, where the position of each pixel in the plane is clear. Used algorithm works, unlike traditional bitmaps with bitmaps working with real numbers, where a step in the direction of axes $x$ or $y$ is not always constant. That is why the algorithm is divided into two steps as was mentioned above.

This method only prevents the incorrect triangulation creation (verification based also on knowledge described in [20]), but does not propose appropriate triangulation. Algorithm is implemented in following steps (Fig. 6):

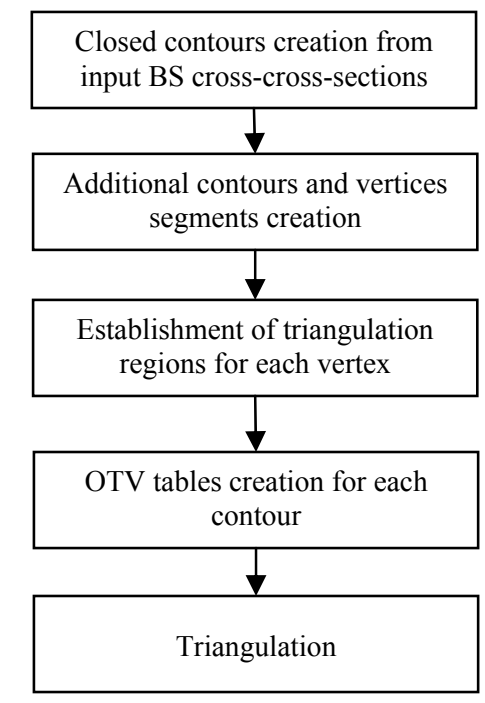

Fig. 6 Implemented modelling algorithm

For each vertex $U$ of contour will be defined the optimal triangulation vertex $V$. These pairs of vertexes $(U, V)$, where $U$ and $V$ lie on adjacent contours, are used for triangulation (the last algorithm step) to design optimal triangulation edges that will faithfully represent the object surface (see Fig. 5). Pairs optimality is defined in the smallest possible distance from the vertex $U$ to $V$ vertex (in space) finding.

For each vertex $U$ is a table of vertexes $V$ generated ordered by their distance (OTV table). This step in the reconstruction algorithm takes the most time. The heuristic approach of optimal pairs search is used during the implementation. This approach generates not the best pairs in some cases and some procedures based on [20] are used for optimization.

The above-described 3D modelling process result is not just BS 3D displaying (see Fig. 7), but also the BS handling possibility along with current views chaotic attractor with BS. Thereby that chaotic attractor never intersect BS confirms that BS is actually a surface separating from each other chaotic attractor and SLC.
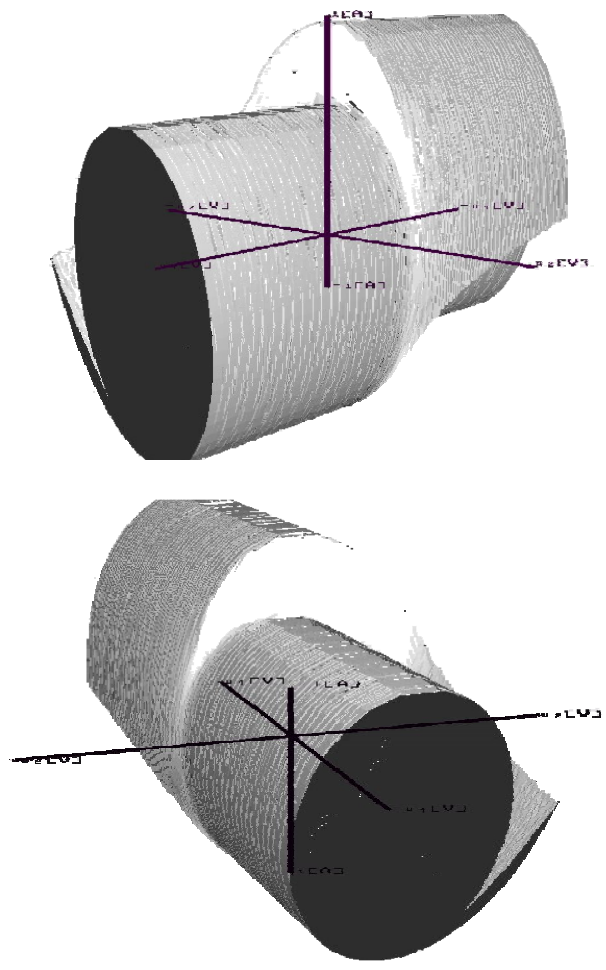

Fig. 7 Final 3D model of BS of Chua's circuit (two different views)

\section{CONCLUSIONS}

The proposed 3D modelling of BS represents further progress in its display in case that the BS morphology is still complicated. It is possible that changes of chaotic attractor, from double scroll to triple or multi scroll, will give rise to more "sharp teeth" of BS and it will be even more morphologically complicated. Described algorithm after some modifications enables its visualization and thus the 3D state space intuitive visualization.

Calculation of BS cross-sections of Chua's circuit as well as BS reconstruction and modelling are timeconsuming processes. Therefore, when these activities in the future will be calculated using GPGPU technology [21], the author promises a significant reduction of calculation time by optimizing process distribution [22][23]. 


\section{ACKNOWLEDGMENTS}

This work is the result of the project implementation: Center of Information and Communication Technologies for Knowledge Systems (ITMS project code: 26220120020) supported by the Research \& Development Operational Program funded by the ERDF.

\section{REFERENCES}

[1] ŠPÁNY, V. - GALAJDA, P. - GUZAN, M.: The State Space Mystery in Multiple-Valued Logic Circuit with Load Plane - Part I. In: Acta Electrotechnica et Informatica, Vol. 1, No. 1, 2001, pp. 17-22, ISSN 13358243.

[2] GALAJDA, P. - GUZAN, M. - ŠPÁNY, V.: The State Space Mystery with Negative Load. Radioengineering, Vol. 8, No. 2, 1999, pp. 2-7.

[3] GUZAN, M. - ŠPÁNY, V. - GALAJDA, P. - BUČKO, R.: Visualization of Boundary Surface Multiple-Valued Logic Memory Using Matlab, Conf. TCB 2010, http://posterus.sk/tcb2010cd/page2/full_text/ 032_Guzan.pdf

[4] ŠPÁNY, V.: The Analysis of a One Tunnel Diode Binary, Procedings IEEE, Vol. 55, june 1967, pp. 10891090.

[5] ŠPÁNY, V.: The Simple VA Characteristic Approximation and the Analysis of the Tunnel Diode Binary, Procedings Letters 1967, pp. 1641-1643.

[6] ŠPÁNY, V.: Graphical Solution of the Nonlinear Circuit with the Help of the M-Dimensional State Space (in Slovak). Electrical Engineering, No. 4, 1969, pp. 233248.

[7] ŠPÁNY, V.: Boundary Surface and Model of Sequential Circuit (in Slovak), Proceedings of scientific papers VST in Košice, 1985, pp. 103-127.

[8] ŠPÁNY, V. - PIVKA, L.: Dynamic Properties of FlipFlop Sensors, Electrical Engineering, 1996, No. 7-8, pp. 169-178.

[9] ŠPÁNY, V. - PIVKA, L. - GALAJDA, P. - GUZAN, M. - KALAKAJ, Ś.: The Calculation of Symmetrizing Voltage in Flip-Flop Sensors, Conf. Radioelektronika 1994, pp. 22-27.

[10] ŠPÁNY, V.: Multistable Systems and Special Surfaces of Multimensional State Space (in Slovak), Electrical Engineering, 1982, No. 7, pp. 551-556.

[11] ŠPÁNY, V. - PIVKA, L.: Boundary Surfaces in Sequential Circuits. International Journal of Circuit Theory and Applications, Vol. 18, 1990, No. 4, pp. 349360.

[12] ŠPÁNY, V. - GALAJDA, P. - GUZAN, M.: Boundary Surfaces of One-port Memories. 5th International Conference Tesla Millennium, Beograd, 1996, October 15-18, pp. 130-137.

[13] MATSUMOTO, T. - CHUA, L. - KOMURO, M.: The Double Scroll, IEEE Transaction on Circuits and Systems, Vol. CAS - 32, No. 8, August 1985, pp. 798818.
[14] ŠPÁNY, V. - GALAJDA, P. - GUZAN, M. - PIVKA, L., OLEJÁR, M.: Chua's Singularities: Great Miracle in Circuit Theory. International Journal of Bifurcation and Chaos, Vol. 20, No. 10, 2010, pp. 2993-3006.

[15] MOLČAN, M.: A Chaos Visualisation, Diploma thesis, DCI FEEI TU Košice, 2009.

[16] LORENSEN, W. E. - CLINE, H. E.: Marching Cubes: a High Resolution 3D Surface Construction Algorithm. Computer Graphics, Issue 4, 1987, pp. 163-169.

[17] ELVINS, T. T.: A Survey of Algorithms for Volume Visualization. Computer Graphics, Vol. 26, Issue 3, 1992, pp. 194-201.

[18] SOBOTA, B. - STRAKA, M. - VAVREK, M.: The Preparation of Contribution of 3D Model Input Using Triangulation, MOSMIC 2007 - Modelling and Simulation in Management, Informatics and Control, 15. - 16.10.2007, Žilina, Faculty of Management Science and Informatics, University of Žilina, 2007, pp. 21-26, ISBN 978-80-8070-807-8.

[19] SOBOTA, B. - STRAKA, M. - PERHÁČ, J.: Some Problems of Virtual Object Modeling for Virtual Reality Applications, Journal of Information, Control and Management Systems, Vol. 6, 1, 2008, pp. 105-112, ISSN 1336-1716.

[20] VÝROST, M. - KOREČKO, Š. - HUDÁK, Š.: Concepts of Refinement and Simulation in BPA and BMethod, Journal of Computer Science and Control Systems, 2, 2, 2009, pp. 74-79, ISSN 1844-6043.

[21] SOBOTA, B. - PERHÁČ, J. - SZABÓ, Cs.: Distributed GPGPU - New Trends, Grid Computing for Complex Problem 2009, Bratislava, 26.-28.10.2009, Bratislava, Institute for Informatics Slovak Academy of Sciences, 2009, 5, pp. 138-145, ISBN 978-80-970145-1-3.

[22] TOMÁŠEK, M.: Types for Calculus of Mobile Code Applications. Acta Electrotechnica et Informatica, Vol. 7, No. 4, ISSN 1335-8243, pp. 68-73, Košice, 2007.

[23] TOMÁŠEK, M.: Controlling Communication and Mobility by Types with Behavioral Scheme. Acta Polytechnica Hungarica, Vol. 5, No. 4, ISSN 17858860, pp. 29-40, Budapest, 2008.

Received September 8, 2010, accepted February 15, 2011

\section{BIOGRAPHY}

Branislav Sobota was born on 22.05.1967. In 1990 he graduated (MSc.) with honours at the Department of Computers and Informatics of the FEEI at Technical University in Košice. He defended his PhD. in 1999 and habilitation thesis in 2008 in the field of virtual reality and computer graphics. He is working as an associate professor at the Department of Computers and Informatics. His scientific research is focusing on computer graphics, virtual reality, modelling and simulation and parallel computing. 